\title{
Correction to: The evaluation of tinnitus and auditory brainstem response in benign paroxysmal positional vertigo accompanied by tinnitus
}

\author{
Ece Kocabaş ${ }^{1} \cdot$ Ahmet Kutluhan $^{2} \cdot$ Banu Müjdeci $^{3}$ (D)
}

Published online: 20 November 2020

○) Springer-Verlag GmbH Germany, part of Springer Nature 2020

\section{Correction to: European Archives of Oto-Rhino-Laryngology https://doi.org/10.1007/s00405-020-06413-w}

Corrections proposed to be made in the final version were transferred by mistake to the online article. The original article has been corrected.

In the original publication of the article, under the abstract, the following sentence "...The difference between the $\mathrm{V}$. wave $=$ false Wave $\mathrm{V}=$ true latency at high rate and $\mathrm{V}$. wave $=$ false Wave $\mathrm{V}=$ true latency ..." was published incorrectly. The correct sentence should read as "... The difference between the Wave V latency at high rate and Wave V latency...".

In addition, the name of the department in affiliation 2 was incorrect. The correct affiliation is, "Faculty of Medicine, Department of Otorhinolaryngology, Ankara Yıldırım Beyazıt University, Ankara, Turkey”.

The original article can be found online at https://doi.org/10.1007/ s00405-020-06413-w.

Banu Müjdeci

banumujdeci@gmail.com

Ece Kocabaş

ecekaraboga@hotmail.com

Ahmet Kutluhan

ahkutluhan@hotmail.com

1 Department of Auditory Rehabilitation, Su Special Training and Rehabilitation Center, Ankara, Turkey

2 Faculty of Medicine, Department of Otorhinolaryngology, Ankara Yıldırım Beyazıt University, Ankara, Turkey

3 Faculty of Health Science, Department of Audiology, Ankara Yıldırım Beyazıt University, Ankara, Turkey
Under the introduction section, the following sentence “...vestibular system have been identified in the internal auditory canal, core level, and Hearing cortex $=$ False Auditory cortex $=$ True..." was published incorrectly. The correct sentence should read as "...vestibular system have been identified in the internal auditory canal, core level, and auditory cortex...".

In the first sentence of ABR section, the term "Neorosoft" was incorrect. The correct term should read as "Neurosoft".

Under the ABR section, the following sentence "...Absolute latency on wave $=$ false Wave $=$ true waves I, III, and $\mathrm{V}=$ false Waves I, III and $\mathrm{V}=$ true and interpeak latency waves I-III, III-V and I-V = False Waves I-III, III-V and $\mathrm{I}-\mathrm{V}=$ True and $\mathrm{I}-\mathrm{V}$ were recorded at low and high rates..." was published incorrectly. The correct sentence should read as “...Absolute latency on Waves I, III, and V and interpeak latency Waves I-III, III-V and I-V were recorded at low and high rates...". 
Table 1 Low and high rate ABR findings of the affected ears of the individuals in Group

Table 2 Low and high rate ABR findings of the affected ears of Group II individuals

Table 3 Comparison of the affected ear and control group ABR findings of patients with BPPV

\begin{tabular}{|c|c|c|c|c|}
\hline ABR latency (ms) & $\begin{array}{l}\text { Group I }(n=30) \text { affected } \\
\text { ear }(9.1 / \mathrm{s}) \\
\text { Mean } \pm \text { SD }(\min -\max )\end{array}$ & $\begin{array}{l}\text { Group I }(n=30) \text { affected } \\
\text { ear }(49.1 / \mathrm{s}) \\
\text { Mean } \pm \text { SD }(\min -\max )\end{array}$ & $\begin{array}{l}\text { Mean difference } \\
\text { between } 9.1 / \mathrm{s} \text { and } \\
49.1 / \mathrm{s}\end{array}$ & $p^{*}$ \\
\hline Wave I & $1.53 \pm 0.13(1.16-1.77)$ & $1.74 \pm 0.18(1.43-1.98)$ & 0.21 & 0.000 \\
\hline Wave III & $3.56 \pm 0.16(3.20-3.86)$ & $3.76 \pm 0.19(3.44-4.01)$ & 0.20 & 0.000 \\
\hline Wave V & $5.50 \pm 0.20(5.16-5.90)$ & $5.84 \pm 0.25(5.50-6.11)$ & 0.34 & 0.000 \\
\hline I-III Interwave & $2.02 \pm 0.13(1.72-2.41)$ & $2.01 \pm 0.21(1.56-2.67)$ & 0.01 & 0.822 \\
\hline III-V Interwave & $1.94 \pm 0.21(1.61-2.35)$ & $2.08 \pm 0.22(1.61-2.49)$ & 0.14 & 0.001 \\
\hline I-V Interwave & $3.95 \pm 0.18(3.55-4.37)$ & $4.08 \pm 0.24(3.39-4.86)$ & 0.13 & 0.015 \\
\hline
\end{tabular}

*Paired-samples $t$-test

\begin{tabular}{lllll}
\hline ABR latency (ms) & $\begin{array}{l}\text { Group II }(n=30) \\
\text { affected ear }(9.1 / \mathrm{s}) \\
\text { Mean } \pm \text { SD (min-max) }\end{array}$ & $\begin{array}{l}\text { Group II }(n=30) \\
\text { affected ear }(49.1 / \mathrm{s}) \\
\text { Mean } \pm \text { SD }(\min -\max )\end{array}$ & $\begin{array}{l}\text { Mean difference } \\
\text { between 9.1/s and } \\
49.1 / \mathrm{s}\end{array}$ & $p^{*}$ \\
\hline Wave I & $1.59 \pm 0.16(1.24-1.98)$ & $1.75 \pm 0.18(1.46-2.03)$ & 0.16 & 0.000 \\
Wave III & $3.62 \pm 0.20(3.20-3.97)$ & $3.86 \pm 0.42(3.49-5.93)$ & 0.24 & 0.007 \\
Wave V & $5.55 \pm 0.21(5.27-6.00)$ & $5.89 \pm 0.25(5.53-6.18)$ & 0.34 & 0.000 \\
I-III Interwave & $2.03 \pm 0.22(1.51-2.54)$ & $2.02 \pm 0.24(1.56-2.59)$ & 0.01 & 0.709 \\
III-V Interwave & $1.93 \pm 0.23(1.50-2.43)$ & $2.11 \pm 0.24(1.82-2.72)$ & 0.18 & 0.000 \\
I-V Interwave & $3.93 \pm 0.27(3.47-4.50)$ & $4.11 \pm 0.28(3.43-4.76)$ & 0.18 & 0.000 \\
\hline
\end{tabular}

*Paired-samples $t$-test

\begin{tabular}{llll}
\hline ABR latency $(\mathrm{ms})$ & $\begin{array}{l}\text { Groups I and II }(n=60) \text { affected } \\
\text { ear } \\
\text { Mean } \pm \text { SD }(\text { min-max })\end{array}$ & $\begin{array}{l}\text { Control }(n=30) \\
\text { Mean } \pm \text { SD }(\text { min-max })\end{array}$ & $p^{*}$ \\
& $1.54 \pm 0.16(1.16-1.98)$ & $1.54 \pm 0.14(1.19-1.91)$ & 0.724 \\
9/s Wave I & $3.59 \pm 0.18(3.20-3.97)$ & $3.55 \pm 0.15(3.23-3.89)$ & 0.204 \\
9/s Wave III & $5.52 \pm 0.21(5.16-6.00)$ & $5.54 \pm 0.21(5.13-6.03)$ & 0.769 \\
9/s Wave V & $2.03 \pm 0.18(1.51-2.54)$ & $2.00 \pm 0.17(1.61-2.46)$ & 0.469 \\
9/s I-III Interwave & $1.93 \pm 0.22(1.50-2.43)$ & $1.98 \pm 0.17(1.46-2.41)$ & 0.152 \\
9/s III-V Interwave & $3.94 \pm 0.24(3.47-4.50)$ & $4.03 \pm 0.22(3.47-4.50)$ & 0.160 \\
9/s I-V Interwave & $1.74 \pm 0.18(1.43-2.28)$ & $1.67 \pm 0.24(1.35-1.96)$ & 0.530 \\
49/s Wave I & $3.81 \pm 0.32(3.44-5.93)$ & $3.70 \pm 0.29(3.31-4.10)$ & 0.046 \\
49/s Wave III & $5.86 \pm 0.25(5.50-6.18)$ & $5.76 \pm 0.31(5.40-6.30)$ & 0.105 \\
49/s Wave V & $2.02 \pm 0.22(1.56-2.67)$ & $2.10 \pm 0.19(1.61-2.59)$ & 0.178 \\
49/s I-III Interwave & $2.10 \pm 0.23(1.61-2.72)$ & $2.10 \pm 0.30(1.75-2.62)$ & 0.771 \\
49/s III-V Interwave & $4.09 \pm 0.26(3.39-4.76)$ & $4.08 \pm 0.23(3.65-4.50)$ & 0.946 \\
49/s I-V Interwave & &
\end{tabular}

*Independent-samples $t$ test
The value of ABR latency (ms) was published incorrectly in Tables 1, 2 and 3. The correct version of tables are provided below.
Publisher's Note Springer Nature remains neutral with regard to jurisdictional claims in published maps and institutional affiliations. 